\title{
Nonlinear grating interaction in photorefractive Bi12SiO20
}

\author{
Buchhave, Preben; Andersen, Peter E.; Petersen, Paul Michael; Vasnetsov, Mikhail
}

Published in:

Applied Physics Letters

Link to article, DOI:

10.1063/1.114190

Publication date:

1995

Document Version

Publisher's PDF, also known as Version of record

Link back to DTU Orbit

Citation (APA):

Buchhave, P., Andersen, P. E., Petersen, P. M., \& Vasnetsov, M. (1995). Nonlinear grating interaction in photorefractive Bi12SiO20. Applied Physics Letters, 66(7), 792-794. https://doi.org/10.1063/1.114190

\section{General rights}

Copyright and moral rights for the publications made accessible in the public portal are retained by the authors and/or other copyright owners and it is a condition of accessing publications that users recognise and abide by the legal requirements associated with these rights.

- Users may download and print one copy of any publication from the public portal for the purpose of private study or research.

- You may not further distribute the material or use it for any profit-making activity or commercial gain

- You may freely distribute the URL identifying the publication in the public portal

If you believe that this document breaches copyright please contact us providing details, and we will remove access to the work immediately and investigate your claim 


\title{
Nonlinear grating interaction in photorefractive $\mathrm{Bi}_{12} \mathrm{SiO}_{20}$
}

\author{
Preben Buchhave ${ }^{\text {a) }}$ and Peter E. Andersen \\ Physics Department, Technical University of Denmark, DK-2800 Lyngby, Denmark \\ Paul Michael Petersen \\ Department of Optics and Fluid Dynamics, Risb National Research Laboratories, \\ DK-4000 Roskilde, Denmark \\ Mikhail Vasnetsov \\ Institute of Physics, Academy of Sciences of Ukraine, Kiev, Ukraine
}

(Received 2 September 1994; accepted for publication 5 December 1994)

\begin{abstract}
Recently significant crosstalk has been observed in a multibeam experiment in which gratings were previously thought to be independent. In this letter, it is shown that the crosstalk is due to a coherent nonlinear combination of the primary gratings, which causes additional peaks to occur in the diffraction pattern and changes the diffraction efficiency of the primary gratings explaining the apparent crosstalk. It is shown that the effect can be derived from the band transport model when all the terms in the expression for the generation of charge carriers are retained. Results are presented for a configuration consisting of a reference beam and two object beams and show experimental results that confirm the model. (c) 1995 American Institute of Physics.
\end{abstract}

It is well known that under certain conditions the formation of the space-charge field in photorefractive $\mathrm{Bi}_{12} \mathrm{SiO}_{20}$ (BSO) is a highly nonlinear process, and much effort has been spent on the description of the form of the space-charge grating resulting from the intersection of two plane optical waves. In this letter, we demonstrate that when three or more coherent beams are incident on a BSO crystal the highly nonlinear process of space-charge formation creates grating frequency components that are combinations of the primary optical grating frequencies. The effect may be derived from the band transport model by retaining all the terms in the expression for the generation of space charge carriers corresponding to all the optical interference patterns. Moreover, these nonlinear coherent combinations of gratings strongly affect the strengths of the primary gratings, and this fact has important consequences for the understanding of the diffraction process in BSO. The effect explains an unexpectedly large crosstalk between neighboring gratings written in BSO by one reference beam and multiple object beams in a configuration, which was previously thought to be without crosstalk. $^{1}$

The experimental situation is illustrated in Fig. 1. Two plane wave object beams $(514 \mathrm{~nm}), \mathrm{O}_{1}$ and $\mathrm{O}_{2}$, are incident onto a BSO crystal at a small angle to the $z$ axis, which is normal to the crystal face. A plane wave reference beam $R$ is incident at approximately the same angle to the other side of the $z$ axis. The crystal operates in the diffusion regime (no externally applied electric field) and the angle between the object beams and the reference beam is selected to allow efficient holographic grating formation between $\mathrm{O}_{1}$ and $R$ and between $\mathrm{O}_{2}$ and $R$. These gratings we denote as $\mathrm{G}_{01}$ and $\mathrm{G}_{02}$, respectively. The long wave optical interference pattern formed between the two object beams is incapable of writing an index grating, $\mathrm{G}_{12}$, in the diffusion regime.

$\mathrm{O}_{2}$ may be phase modulated by means of a piezomirror. By controlling the amplitude of the sinusoidal phase modu-

${ }^{a)}$ Electronic mail: pbu@mips.fys.dth.dk lation it is possible to control the strength of the grating associated with the phase modulated object beam, and at a certain amplitude the grating may be completely erased. This scheme allows an analog control of the strength of the interconnect. $^{2}$ According to Ref. 2, the advantage of this method is that the total intensity of light incident on the crystal is unchanged, and thus one grating may be modulated or switched off without affecting the diffraction efficiencies of the unmodulated gratings.

This model neglects the possibility of energy transfer between the object beams by diffractive coupling through the reference beam. However, the beam coupling in BSO in the diffusion regime is usually small; Marrakchi et al. ${ }^{3}$ in similar experiments show the beam coupling to be of the order of $1 \%$.

Experiments ${ }^{1}$ have nonetheless shown that under certain conditions a much greater crosstalk is found than would be expected from the simple description outlined in Ref. 2. We define crosstalk $\Delta \eta$ as the relative change in diffraction efficiency of grating $\mathrm{G}_{01}$ as a result of phase modulating beam $\mathrm{O}_{2}$. Using this definition it was found that $\Delta \eta$ could assume values between $+45 \%$ and $-5 \%$ at particular values of the ratio $\beta$ between the reference beam intensity $I_{0}$ and the sum of the two object beam intensities, $I_{1}+I_{2}$, even though the diffraction angles for the various gratings are well outside the Bragg range, and they are thus expected to be independent. Figure 2 shows a typical example of $\Delta \eta$ as a function

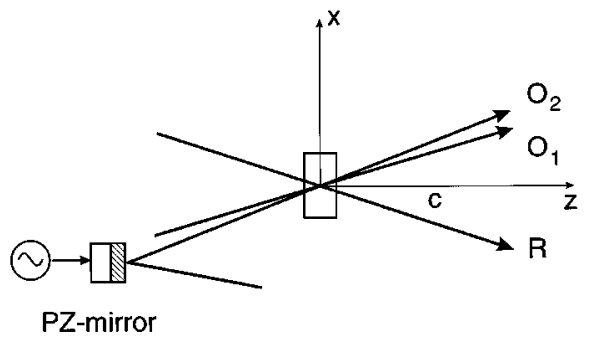

FIG. 1. Multiple-wave interaction in BSO. $\mathrm{R}$-reference beam. $\mathrm{O}_{1}$ and $\mathrm{O}_{2}$ object beams. 


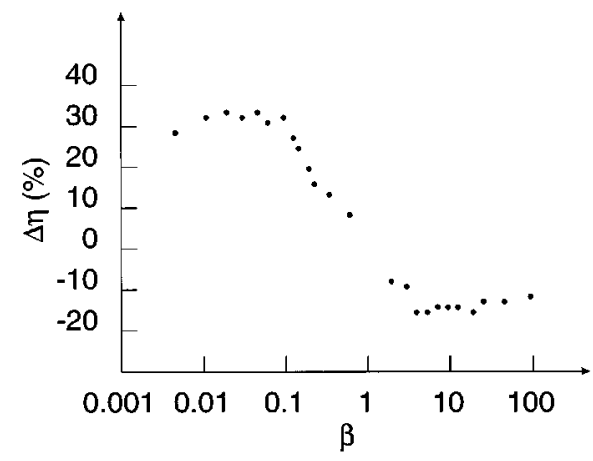

FIG. 2. Crosstalk between object beams as a function of beam ratio $\beta$; object beam $\mathrm{O}_{2}$ phase modulated.

of $\beta$. The crosstalk is found to depend primarily on $\beta$, while being nearly independent of the angle separating the object beams. The maximum crosstalk occurs at a value of $\beta$ of about 0.1 , while it diminishes for both very small and very large $\beta$. Other experiments show that the crosstalk is also independent of the absolute magnitude of the beam intensities. The magnitude of the crosstalk is quite clearly much greater than can be explained by the mechanisms outlined above.

The crosstalk described above can be explained by the nonlinear coupling between multiple gratings written in BSO. In our experimental situation with two primary gratings of nearly the same grating frequencies (grating period 1.081 and $1.074 \mu \mathrm{m}$ with an angular difference of $1.7 \mathrm{mrad}$ ), additional grating components are formed with frequencies deviating from those of the primary gratings by multiples of their difference frequency. Note that in this process the grat- ing $\mathrm{G}_{12}$ between the two object beams is not physically present - a fact we have confirmed experimentally.

When an external voltage is applied to the crystal (drift regime) the same nonlinear interaction between the gratings takes place, but in this case the low frequency grating is physically present in the crystal.

The space-charge field in a photorefractive BSO crystal may be written: ${ }^{4}$

$$
E=\frac{j}{e \mu n}+\frac{T}{e} \frac{1}{n} \frac{\partial n}{\partial x}+\frac{p I}{e \mu n},
$$

where $n$ is the photocarrier density, $e$ the electron charge, $\mu$ the photocarrier mobility, $j$ the Ohmic current density, $T$ the temperature in energy units, $I$ the light intensity, and $p$ the photovoltaic constant. In BSO we may neglect the photovoltaic effect.

We write the photogeneration as a sum of three cosine functions corresponding to the three primary optical gratings:

$$
\begin{aligned}
f= & \left(d+s I_{t}\right)\left\{1+M_{01} \cos \left[K_{01} x+\phi_{01}(z)\right]\right. \\
& +M_{02} \cos \left[K_{02} x+\phi_{02}(z)\right] \\
& \left.+M_{12} \cos \left[K_{12} x+\phi_{12}(z)\right]\right\}
\end{aligned}
$$

where $d$ is the dark current, $s I_{t}$ is the rate of generation of carriers from the total beam intensity $I_{t}=I_{1}+I_{2}+I_{0}, K_{i j}$ $(i, j=0,1,2)$ are the grating constants, and $\phi_{i j}(i, j=0,1,2)$ are phase terms, which we may set to zero. $M_{i j}(i, j=0,1,2)$ are the modulation depths of the gratings: $M_{i j}=m_{i j} /\left(1+\sigma_{d} / \sigma_{p}\right)$, where $\sigma_{d} / \sigma_{p}$ is the ratio of the dark- and photoconductivities and $m_{i j}$ the optical intensity modulation, $m_{i j}=2 \sqrt{I_{i} I_{j}} / I_{t}$.

With no applied field (diffusion) the solution is

$$
E=-\frac{D}{\mu} \frac{M_{01} K_{01} \sin K_{01} x+M_{02} K_{02} \sin K_{02} x+M_{12} K_{12} \sin K_{12} x}{1+M_{01} \cos K_{01} x+M_{02} \cos K_{02} x+M_{12} \cos K_{12} x} .
$$

This is the conventional solution as given in Ref. 4, but all three optical modulation terms in Eq. (2) have been retained. The diffusion coefficient $D$ is, of course, the same for all gratings, but the diffusion field is not: $E_{D, i j}=(D / \mu) K_{i j}$. Since $K_{12} \ll K_{01}, K_{02}$ we have $E_{D, 12} \ll E_{D, 01}, E_{D, 02}$. As a consequence we can write the space-charge field as a sum of the two primary gratings divided by a quantity containing all three optical interference gratings:

$$
E=-\frac{D}{\mu} \frac{M_{01} K_{01} \sin K_{01} x+M_{02} K_{02} \sin K_{02} x}{1+M_{01} \cos K_{01} x+M_{02} \cos K_{02} x+M_{12} \cos K_{12} x} .
$$

Note, that the optical interference pattern between the two object beams is still present in the denominator as a large term because the modulation $M_{12}$ may be close to unity, even when the terms due to the interference between the object beams and the reference beam may be small.

In the drift regime we may no longer neglect the primary grating between $\mathrm{O}_{1}$ and $\mathrm{O}_{2}$. However, the terms in the denominator are unaltered:

$$
E=\frac{j}{e \mu\langle n\rangle} \frac{1}{1+M_{01} \cos K_{01} x+M_{02} \cos K_{02} x+M_{12} \cos K_{12} x},
$$

where $\langle n\rangle$ is the mean electron density.

The diffraction efficiency now follows in the usual way from the relation between space-charge field and refractive index. ${ }^{4}$ To see the frequency contents of the space-charge field we perform a numerical Fourier transform of the expressions Eqs. (4) and (5). The resulting spectra are shown in 

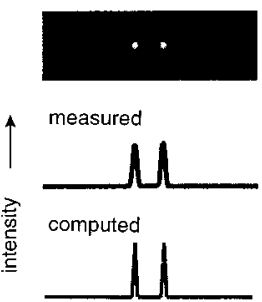

b)
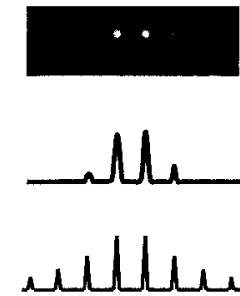

position $\longrightarrow$ c)
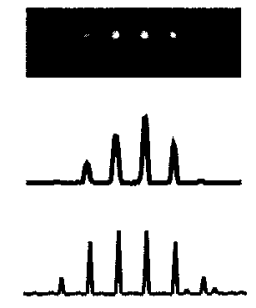

FIG. 3. Computed spectrum of space-charge field and measured diffraction pattern for: (a) Linear superposition of the two primary gratings, diffusion regime $(\beta \gg 1)$. (b) Nonlinear interaction, diffusion regime $(\beta \ll 1)$. (c) Nonlinear interaction, drift regime $(\beta \ll 1)$.

Fig. 3 in the lower line. The upper part shows the diffraction pattern of the reference beam with the object beams turned off. We first consider the diffusion regime.

Strong reference beam $(\beta \gg 1)$ : We find $M_{01}, M_{02} \ll 1$, but also $M_{12} \ll M_{01}, M_{02}$. Thus the terms in the denominator of (4) are much smaller than unity and the space-charge field is a linear superposition of the two high frequency primary gratings [Fig. 3(a)]:

$$
E=-\frac{D}{\mu}\left(M_{01} K_{01} \sin K_{01} x+M_{02} K_{02} \sin K_{02} x\right) \text {. }
$$

The measured diffraction pattern confirms the presence of only two gratings. The same situation may also occur if all the optical interference gratings are weakly modulated, e.g., due to strong background illumination.

Weak reference beam $(\beta \ll 1)$ : Again we find $M_{01}, M_{02} \ll 1$, but now $M_{12}$ is nearly unity. We can no longer disregard the term $M_{12} \cos K_{12} x$ in the denominator, and although the low frequency space-charge grating does not exist physically in the crystal, the low grating frequency shows up in mixing products of the two primary gratings with difference frequencies equal to multiples of the low frequency modulation [Fig. 3(b)].

In the numerical example we have used an optical modulation depth of 0.9. At high optical modulation depths the expression for the space-charge field becomes very unstable with extremely high values for the field strength and strong gradients due to the near singularity in the denominator. However, the real space-charge field strength and the steepness of the gradients are limited by physical processes not included in the above model. We have arbitrarily reduced the grating field strengths by using a value of $M_{12}$ somewhat below unity. The important result here, however, is that strong gratings are formed at new frequencies separated from the primary grating frequencies by multiples of the difference frequency $f_{12}=K_{12} / 2 \pi$.

Note: This effect is not due to higher harmonics of the primary gratings; these would be located far to right of the spots shown in Fig. 3. A possible grating $G_{12}$ formed between the two object beams would be far off scale to the left. However, when probing the crystal with a He-Ne laser at an angle corresponding to the Bragg angle for grating $G_{12}$ we

were unable to detect any diffraction from such a grating. We thus conclude that only the two primary gratings and combination gratings created by the nonlinear form of the spacecharge field are present in the crystal.

In intermediate cases $(\beta \cong 1)$ all three modulation depths must be taken into consideration. The distribution of mixing components is now different, but the spectrum is still dominated by grating terms separated from the primary gratings by multiples of the difference frequency.

Drift case: The terms in the denominator of (5) are still present, and again additional gratings are formed. The strengths of these gratings depend on the details of the intensity modulation. Figure $3(\mathrm{c})$ shows the spectrum and the measured diffraction pattern for $\beta \cong 1$. Probing at the Bragg angle for grating $G_{12}$ now shows the presence of a low frequency grating in addition to the gratings formed with the reference beam.

The form of the diffraction pattern corresponds well to the strengths of the Fourier components of the space-charge field [Eqs. (4) and (5)]. At the same time the drop in the diffraction efficiency of the primary grating $G_{01}$ occurring after turning off the phase modulation agrees with the measured crosstalk. We may describe the influence from the additional gratings as interference terms, which modify the strengths of the primary gratings in the three coupled wave equations describing the diffraction from gratings $G_{01}$ and $G_{02}$. This change in grating strength leads to the change in diffraction efficiency, which is measured as a crosstalk in Ref. 1.

We have shown that nonlinear superposition of multiple gratings may occur in $\mathrm{B}_{12} \mathrm{SiO}_{20}$ both in the diffusion regime and in the drift regime. The effect is due to the nonlinear form of the space-charge field. In a three-beam interaction with one reference beam and two closely situated object beams additional gratings are formed at frequencies offset from the two primary ones by multiples of their difference frequency. In the diffusion regime, this interaction occurs even though the optical interference pattern between the two object beams does not induce a grating, as its frequency is too low. In the drift regime a grating caused by the interference between the two object beams is present and additional gratings occur again. In this case the effect is even stronger.

As all the gratings are coherent the diffraction efficiencies of the primary gratings are also affected. This effect accounts satisfactorily for the unexpectedly large crosstalk occurring in dynamic optical interconnects using phase modulation of the writing beams to control the grating strengths.

${ }^{1}$ P. E. Andersen, P. M. Petersen, and P. Buchhave, Appl. Phys. Lett. 65, 271 (1994)

${ }^{2}$ A. Marrakchi, W. M. Hubbard, S. F. Habiby, and J. S. Patel, Opt. Eng. 29, 215 (1990).

${ }^{3}$ A. Marrakchi, J. P. Huignard, and P. Günter, Appl. Phys. 24, 131 (1981).

${ }^{4}$ N. V. Kukhtarev, V. B. Markov, S. G. Odulov, M. S. Soskin, and V. L. Vinetskii, Ferroelectrics 22, 949 (1979). 